\title{
Research on Intelligent Clothing Blemishes Detection System and Application Based on Cloud Computing
}

\author{
Yihong Zhang ${ }^{1,2, *}$, Bao Zong ${ }^{2}$, Xin Ruan ${ }^{2}$, Lifeng $\mathrm{Shi}^{2}$ and Xiang $\mathrm{Li}^{2}$ \\ ${ }^{1}$ Engineering Research Center of Digitized Textile and Fashion Technology, Ministry of Education, Shanghai, China \\ ${ }^{2}$ College of Information Science and Technology, DongHua University, Shanghai 201620, China \\ ${ }^{*}$ Corresponding author
}

\begin{abstract}
This paper research the clothing blemishes detection theory based on smart clothing support robots, and proposes a clothing detection system based on smart clothing support robot of multi-objective collaborative intelligence; and proposes a clothing quality inspection method based on multifeatures collaboration and multi-source image information fusion to develop a clothing quality inspection prototype system for smart clothes support robots; and set up a smart clothes support robot's clothing blemishes inspection platform based on cloud computing, through the prototype system to solve the key technologies of its application, effectively reduce the companies' cost of clothing blemishes inspection, and lay the theoretical and application foundation for intelligent monitoring of clothing quality.
\end{abstract}

Keywords-blemishes detection; smart clothing; cloud platform; deep learning; image processing

\section{INTRODUCTION}

This paper designed an information fiber sensor for the 6DOF smart hanger application, and placed them in three appropriate positions on the body, shoulders, and arm joints of the smart hanger, and detecting the pressure value of the garment to be tested at three different positions after the smart hanger is put on the garment, and feedback to the intelligent adjustment model to allow each arm to adjust to the best position in the shortest time; Throung the multi-sensor information fusion theory, established a multi-branch garment inspection robot multi-objective collaborative intelligent control strategy library, and through the multi-features of clothing images, the collaborative matching and intelligent recognition mathematical model to achieve effective detection of clothing.

This paper research on panorama fusion technology of 3D graphics based on 3D garment image information acquisition of smart garment support robot, and acquire the multi-angle image of clothing to be tested on the smart clothes support to compose panoramas for image processing links; and establish image processing models suitable for apparel fabrics to detect defects in processed graphics, and determine whether there are any kinds of blemishes on the picture or not. And establish an effective neural network model to identify and classify clothing types.

This paper use the distributed storage to storage data, and realize cloud computing for clothing 3D image mass data storage, and use the redundant storage method to ensure the reliability of stored data; And all clothing's 3D image resources are stored in the "cloud" through cloud computing, and these massive data are managed and dispatched by the "cloud", the segmented clothing three-dimensional image are classified and stored by using distributed data storage technology and virtual technology associated it.

\section{THREE-DimENSIONAL CLOTHING IMAGE INFORMATION ACQUISITION AND PROCESSING SYSTEM BASED ON SMART Clothes SupPort OF AIRBAG IMITATING HuMAN BODY}

\section{A. The Establishment of A Mathematical Model Based on 6- Dof Smart Hanger}

Based on the mathematical modeling and simulation of the 6-DOF smart hangers, the mathematical models and expressions of Zhihuan body joints, shoulder joints, and arm joints were obtained to verify the above theoretical results. In this paper, the airbag and the flexible pressure sensor are combined and adaptive fuzzy control is introduced to propose a smart hanger based on the flexible pressure sensor and its mathematical model and control method. The traditional airbag using method is based on the air pressure parameter as feedback control quantity, this kind of method is difficult to form the good control effect under the complicated actual environment, and after adding a new type of graphene flexible pressure sensor (one of the innovations in this paper), the deformation of the target can be detected more directly; A flexible pressure sensor array is attached to the airbag, and the data obtained shows the suitability of clothes and hangers, which is an important criterion for garment testing. In order to obtain the appropriate affiliation and relatively accurate detection process, an adaptive fuzzy control algorithm was added to control the entire process. Through the simulation and experimental results, the fuzzy controller is compared with the traditional PID controller, and the model and the fuzzy PID control method are effective obtained from the results of the simulation results of the two methods.

\section{B. Intelligent Rack Information Detection and Transmission Model Based on Information Fiber Flexible Sensor Network}

The sensor and the computing element are implanted into the arms of the smart hanger, and the topology of the flexible sensor network is constructed; On the basis of studying the kinematics and dynamics model of the clothing detection robot, 
a digital simulation model based on force, pose, speed, acceleration and other multiple information feedback was established to verify the above theoretical results. The project in this research innovatively used a graphene pressure sensor based on a single-layer graphene film. The single-layer graphene is a sandwich structure formed by two outer layers, due to the low degree of bending of the film and the verticality applied. The force is limited in size, and the applied force can be approximated as a vertical force acting on the film surface.

The measurement of the resistance of the two ends of the graphene film designed with the PDMS/graphene/PDMS sandwich structure shows that the resistance is maintained at about $20 \Omega$ in the flat state. If a vertical force is applied to the surface of the graphene film, the shape will be reduced. Changes occur and shape changes occur. After being subjected to external pressure, the graphene film shows a high degree of sensitivity, and the resistance value can reach $100 \mathrm{k} \Omega$ at the moment. Since the steady-state resistance of the graphene is equivalent to $20 \Omega, 100 \mathrm{k} \Omega$ is equivalent to the open circuit, and there will be a period of process of the steady-state after the instant to the large resistance, the time is related to the size of the deformation.

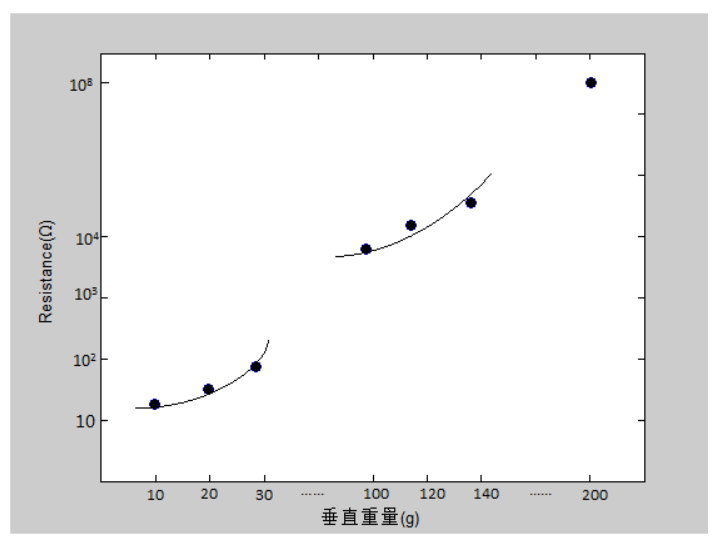

FIGURE I. MEASURED VALUE OF THE GRAPHENE SENSOR AFTER IT HAS STABILIZED UNDER PRESSURE.

TABLE I. PIEZOELECTRIC REACTION OF MONOLAYER GRAPHENE THIN FILMS.

\begin{tabular}{|c|c|c|c|c|c|c|c|}
\hline $\begin{array}{c}\text { Applied } \\
\text { pressure(N) }\end{array}$ & 0.01 & 0.02 & 0.03 & 0.1 & 0.12 & 0.14 & 0.2 \\
\hline $\begin{array}{c}\text { Resistance } \\
(\mathbf{\Omega})\end{array}$ & 30 & 50 & 98 & 2034 & 5069 & 11543 & nan \\
\hline
\end{tabular}

As shown in Figure 1 and Table 1, the weight of the indicated weight is placed on the sandwich of the single-layer graphene pressure sensor and the resistance is measured. The table shows a near-linear relationship in a small range, but due to high sensitivity, the increase of weight make the change in resistance very obvious.

The change in resistance shows that it has the same piezoelectric effect as a normal device. However, because the range of the change is not obvious, we connect the circuit at its periphery to see the voltage changes on both sides, and we can see the piezoelectric characteristics more intuitively. The graphene thin-film pressure sensor is very sensitive to pressure, and the applied pressure instantly exhibits a change in electrical resistance. After the deformation reaches a maximum, the graphene thin film begins to recover. This process is slower than the sudden change in external force. It can be seen that the Athens reaction shown by the flexible pressure sensor under a certain pressure condition is non-linear, but it is not completely irrelevant, and even a near-linear reaction may occur. However, data generated by a single layer or a single sensor may cause problems when used in a smart hanger. For example, a single flexible pressure sensor may exhibit different pressure values depending on where it touches the same pressure when in contact with clothing. This may result in different pressure values. Every time the measured data is more or less different, it is difficult to systematically model it. So this paper uses sensor arrays to achieve. The sensor array detects an area, not a certain position or angle, so that the acquired data will increase the reliability of the model when it is fitted experimentally.

\section{ESTABLISHMENT AND IMPLEMENTATION OF CLOTHING IMAGE ACQUISITION, ANALYSIS, AND SKULL DETECTION THEORY}

\section{A. Collection Of Sample Images of Finished Garment Defects}

- First, the finished garment is vertically suspended by a designed smart hanger, and the high-precision camera is placed on the front.

- In order to ensure the integrity and quality of the defective image extraction, multi-angle shooting of finished garments is adopted. That is, the smart hanger is rotated one week to capture the finished garment image at each angle. Afterwards, image matching can be used. Find out where each image corresponds to.

- This will avoid the inaccuracies caused by using only a single image to find defects, and the noise in the image that interferes with defect detection. Define abbreviations and acronyms the first time they are used in the text, even after they have been defined in the abstract. Abbreviations such as IEEE, SI, MKS, CGS, $\mathrm{sc}$, dc, and rms do not have to be defined. Do not use abbreviations in the title or heads unless they are unavoidable.

\section{B. Image Preprocessing and Defective Image Segmentation}

The image processing algorithm is used to process the images obtained from the collected finished clothes from various angles, such as the graying of the original image, and the contrast of the image is increased through the histogram transformation and the noise is reduced through filtering.

\section{Three-Dimensional Fusion of Clothing Image Information Based on Multi-Source Images}

- Due to the effects of light, motion, and noise, it is highly likely that a single image may not be identified or it may be difficult to extract complete feature information. 
- Three-dimensional fusion of defects from different angles, as much as possible to represent the information, and provide the basis for feature extraction.

\section{Feature Extraction and Recognition and Classification of Blemish Points}

For the blemish image after the fusion of various angles, the feature extraction method is used to extract the information from the defect point image for the final recognition. On the basis of the feature of the extracted image, the classification and recognition system of defect point based on intelligent algorithm is constructed. By extracting the features of the extracted defect points into the classifier, we can get the categories of the defect points through the classifier, so that we can evaluate the quality level of the finished garments according to the types of the defects. Because the defect detection model is relatively mature, the project leader's fabric defect detection model, which is in charge of Hong Kong Polytech University, can solve the problem of defect detection and classification well. Figure 2 shows a schematic diagram of a garment flaw detection system based on cloud computing. Based on the processing and recognition of massive clothing images and defective pictures, we have applied the deep convolutional network to the first attempt to identify and classify the clothing types. In view of the time factor, a large number of clothing defects will be tested in the future. In order to get evidence of a given picture belonging to a particular number class (evidence), we weighted the pixel value of the picture. If this pixel has strong evidence that the picture does not belong to the class, the corresponding weight is negative. Conversely, if the pixel has favorable evidence to support the picture belongs to this class, the weight is positive. The following picture shows the weight of each pixel on a particular number class on the picture that a model has learned. The red represents the negative weight, and the blue represents the positive weight.

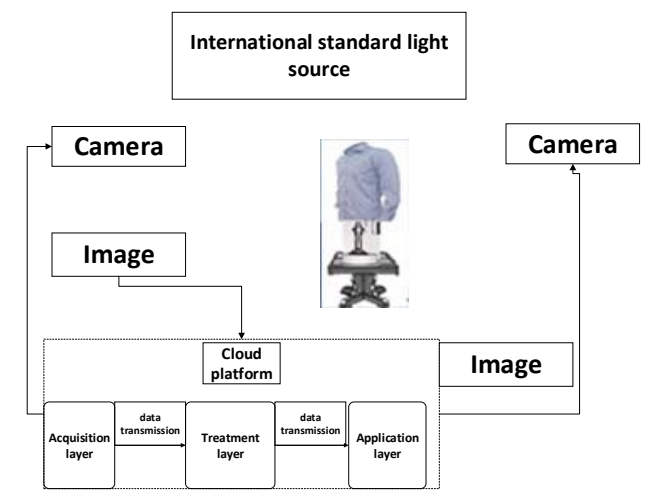

FIGURE II. SKETCH MAP BASED ON CLOUD COMPUTING

We also need to add an additional offset (bias), because the input tends to have some unrelated interference. Therefore, for a given input picture $x$, the evidence that it represents is a digital I can be expressed as

$$
\text { evidence }_{i}=\sum_{j} W_{i, j} x_{j}+b_{i}
$$

$$
y=\operatorname{softmax}(\text { evidence })
$$

The softmax here can be seen as an activation function or a link function, the output of the linear function we define is converted to the format we want, that is, the probability distribution of the 10 numeric classes. Therefore, given a picture, it can be converted into a probability value by the softmax function for the consistency of each number. The softmax function can be defined as:

$$
\begin{gathered}
\operatorname{softmax}(x)=\text { normalize }(\exp (x)) \\
\operatorname{soft}(x)_{i}=\frac{\exp \left(x_{i}\right)}{\sum_{j} \exp \left(x_{j}\right)} \\
y=\operatorname{softmax}(W x+b)
\end{gathered}
$$

The cost function "cross entropy" (cross-entropy) is expressed in the following formula 6.

$$
H_{y^{\prime}}(y)=-\sum_{i} y_{i}^{\prime} \log \left(y_{i}\right)
$$

Among them, $y$ is the probability distribution we predict, the Y'is the actual distribution, and the TensorFlow has a graph that describes the various computing units. It can automatically use the backpropagation algorithm to effectively determine how your variable affects the cost value that you want to minimize. The TensorFlow will then use the selected optimization algorithm to constantly modify the variable to reduce the cost.

TensorFlow is required to minimize cross entropy with a gradient descent algorithm (gradient descent algorithm) at a learning rate of 0.01 . The gradient descent algorithm (gradient descent algorithm) is a simple learning process. TensorFlow only needs to move every variable to the direction of decreasing cost. Figure 18 is a schematic diagram of the model structure of the LeNet5 8 layer CNN.

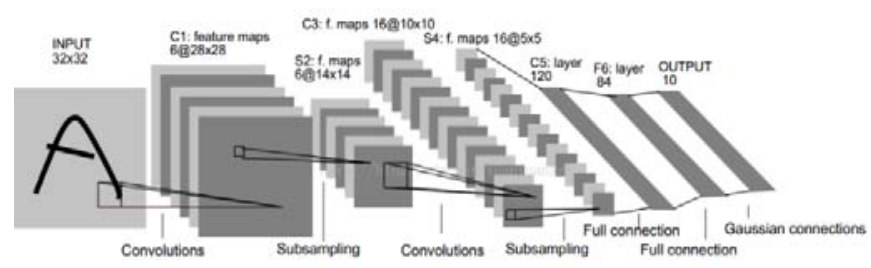

FIGURE III. A SCHEMATIC DIAGRAM OF THE MODEL STRUCTURE OF THE LENET5 8 LAYER CNN

The first layer is a convolution layer. Input is the original image pixel, $32 * 32 * 1$, convolution filter size is $5 * 5$, depth is 6 , do not use all 0 fill, step size is 1 , output size is $28(32-5+1)$ The depth is 6 , the convolution layer has a total of 156 parameters $(5 * 5 * 1 * 6+6$ (offset terms)), and the next layer node matrix has $4704(28 * 28 * 6)$ nodes, each The nodes are connected to $25(5 * 5)$ current layer nodes, so there are 122304 
$(4704 *(25+1))$ connections in the convolution layer. The second layer is the pooled layer, the input is the output of the first layer, the node matrix of 4704 , the filter size is $2 * 2$, the step size is 2 , and the output matrix size is $14 * 14 * 6$. The third layer is the convolutional layer, the input is the output of the second layer, the filter size is $5 * 5$, the depth is 16 , do not use all 0 fill, the step size is 1 , and the output matrix size is $10 * 10 * 16$. The layer contains $2416(5 * 5 * 6 * 16+16)$ parameters and $41600(10 * 10 * 16 *(25+1))$ connections. The fourth layer is the pooled layer, the input is the output of the third layer, the filter size is $2 * 2$, the step size is 2 , and the output proof size is $5 * 5 * 16$. The fifth layer is the full connection layer (or convolutional layer, filter size $5 * 5=$ input size), 400 input nodes, 120 output nodes, $48120(5 * 5 * 16 * 120+120)$ parameters. The sixth layer is a fully connected layer, 120 input nodes, 84 output nodes, $10164(120 * 84+84)$ parameters. The seventh layer is a fully connected layer, 84 input nodes, 10 output nodes, and $850(84 * 10+10)$ parameters. Tips represent the main role of the pooling layer is to prevent over-fitting, In some papers, it is found that the convolution step can be adjusted, so some networks remove the pool layer to speed up the operation.

\section{THE ESTABLISHMENT AND IMPLEMENTATION OF THE Theory of Image Processing Model Based on Cloud COMPUTING PLATFORM}

In this paper, a innovative convolution neural network is used to identify the styles of clothing, which lays a technical foundation and research orientation for the recognition of the defects in the future. Based on the processing and recognition of massive clothing images and defective pictures, we have applied the innovative deep convolutional network to the first attempt to identify and classify the clothing types. In view of the time factor, a large number of clothing defects will be tested in the future

\section{A. Segmentation and Classification of Mass Data of a 3D Image}

The captured clothing three-dimensional image information is segmented using image segmentation technology, and the different parts of the clothing are classified and stored.

\section{B. Massive Data Compression and Transmission of Garment 3D Images}

The method of equal-volume parallel volume compression and transmission((Each volume of data is equal, the compression and transmission processes between the volumes are processed is parallel)) is used to first perform roll-to-roll compression on the classified data((ZIP is used to compress the block data at the same time), and then the transmission is realized on the basis of data compression.

\section{Storage of Mass Data of Clothing 3D Images in Cloud Computing Environment}

Cloud computing has powerful hardware resources and software resources, and can implement distributed parallel processing. It can achieve fast and efficient data processing for $3 \mathrm{D}$ images of clothing. The use of distributed storage for data storage enables cloud computing to store large amounts of 3D images of clothing, and uses redundant storage to ensure the reliability of stored data. Using cloud computing technology to store all 3D image resources in the "cloud" and manage and schedule these massive data by "cloud". The distributed data storage technology and the related virtual technology are used to store and classify the various pieces of data of the segmented clothing three-dimensional image.

\section{Clothing Classification Processing Algorithm Based on Deep Learning}

The process of taking the new image through a good convolution neural network to the bottleneck layer can be regarded as the feature extraction process of the image. In the trained Inception-V3 model, the output of the bottleneck layer can distinguish 1000 classes of images well through a single layer full link layer neural network. Therefore, there is a reason to think that the node vector of the bottleneck layer output can be used as a more streamlined and more expressive feature vector of any image. Thus, the trained neural network can be used to extract the features of the image on the new dataset, then the extracted feature vectors are used as input to train a new single layer fully linked neural network to deal with new classification problems. In general, when the amount of data is sufficient, the effect of migration learning is not as good as retraining. However, the number of training time and training samples required for the migration learning is much smaller than that of the complete training model. The following picture is a sample picture data set of all kinds of clothing collected through image.net.

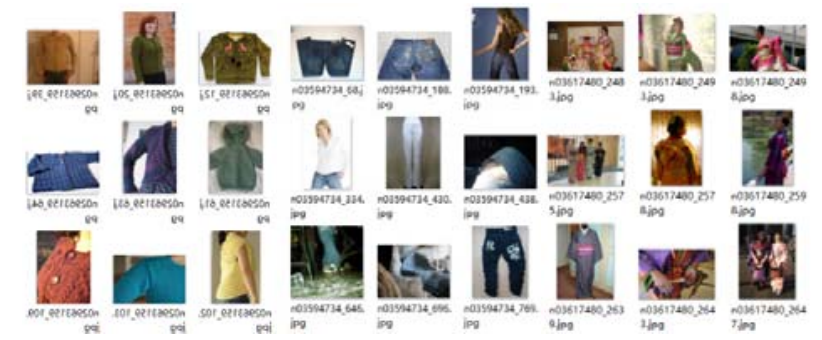

FIGURE IV. VARIOUS GARMENT SAMPLE DATA SETS

After 1000 training, we can reach a $98.9264 \%$ recognition rate. And by the recognition of another group of clothing data groups, we can achieve a $98.6865 \%$ recognition rate by 1000 training, which is sufficient to prove the reliability of the model.

\section{ACKNOWLEDGMENT}

This research work was supported by the China Central University Foundation, Project Number: [18D110408].

\section{REFERENCES}

[1] Yihong Zhang, Xi Jin,Zhijie Wang,Research And Application Of Smart Hanger Based On Airbag Inflation Model and Fuzzy Self-adaptive PID method.,Accepted by CAAI2017.

[2] Yi-hong ZHANG, Xi JIN and Zhi-jie WANG, Research And Application Of Smart Hanger Based On Airbag Inflation Model and Multi-object Collaborative Optimization Algorithm. Accepted by CAEME 2017. 
[3] M. Mao, K.-R. Hao, and Y.-S. Ding, Extracting corresponding-point based on texture synthesis for nearly flat textureless object surface, Mathematical Problems in Engineering, 2015, 1-16.

[4] Y.H.Zhang, X.Jin, Z.J.Wang, A new modified panoramic UAV image stitching model based on the GA-SIFT and adaptive threshold method.

[5] Yihong Zhang, Li Xiang, Fang Wei,Research of garment defect recognition algorithm based on fuzzy Weber local Descriptor and deep belief network.

[6] Y.H.Zhang, X.Jin, Z.J.Wang, Intelligent garment defect detecting model based on deep learning network and cloud computing.

[7] Y.H.Zhang, X.Jin, Z.J.Wang, Intelligent garment style recognition model based on optimal convolutional neural network.

[8] Y.H.Zhang, X.Jin, Z.J.Wang, A new modified panoramic UAV image stitching model based on the GA-SIFT and adaptive threshold method.

[9] Yi-hong Zhang, Chi-wai Kan and Jimmy Kwok-cheong Lam, A study oultraviolet protection properties of $100 \%$ cotton knit fabric: effect of fabric structure, The Journal of The Textile Institute,2014.

[10] Yihong Zhang,Xi Jin,Zhijie Wang,Research And Application Of Smart Hanger Based On Airbag Inflation Model and Fuzzy Self-adaptive PID method.,Accepted by CAAI2017.

[11] Y. Liu, K. R. Hao, Y. S. Ding. Moving Human Posture Recognition Based on Joint Quaternion, J. Donghua University, 2016, 33(5).

[12] Deng L L. Pre-detection Technology of Clothing Image Segmentation Based on GrabCut Algorithm[J]. Wireless Personal Communications, 2017(1):1-12.

[13] Tong L, Wong W K, Kwong C K. Fabric Defect Detection for Apparel Industry: A Nonlocal Sparse Representation Approach[J]. IEEE Access, 2017, 5(99):5947-5964.

[14] Sun G L, Wu X, Peng Q. Part-based clothing image annotation by visual neighbor retrieval[J]. Neurocomputing, 2016, 213:115-124.

[15] Chen M, Ma Y, Song J, et al. Smart Clothing: Connecting Human with Clouds and Big Data for Sustainable Health Monitoring[J]. Mobile Networks \& Applications, 2016, 21(5):825-845.

[16] Jing J, Liu S, Li P, et al. The fabric defect detection based on CIE $\mathrm{L}^{*} \mathrm{a} * \mathrm{~b}^{*}$ color space using 2-D Gabor filter[J]. Journal of the Textile Institute Proceedings \& Abstracts, 2016, 107(10):1305-1313.

[17] Zheng D, Wang L. Multi-scale density detection for yarn-dyed fabrics with deformed repeat patterns[J]. Textile Research Journal, 2016, 87(20).

[18] Lizarraga-Morales R A, Sanchez-Yanez R E, Baeza-Serrato R. Defect detection on patterned fabrics using texture periodicity and the coordinated clusters representation[J]. Textile Research Journal, 2016, 87(15).

[19] Stivanello M E, Vargas S, Roloff M L, et al. Automatic Detection and Classification of Defects in Knitted Fabrics[J]. IEEE Latin America Transactions, 2016, 14(7):3065-3073.

[20] Martinez-Leon M, Lizarraga-Morales R A, Rodriguez-Donate C, et al. Defect Detection on Patterned Fabrics Using Entropy Cues[C]// International Conference on Image and Signal Processing. Springer, Cham, 2016:71-78. 